\title{
Olhares distintos sobre a noção de estabilidade e mudança no desempenho da coordenação motora grossa
}

\author{
Luciano BASSO* \\ Cleverton J osé Farias de SOUZA* \\ Ulysses Okada de ARAÚJ O* \\ Flávio Henrique BASTOS* \\ Thales Teixeira BIANCHI ${ }^{* *}$ \\ Cássio Miranda MEIRA J ÚNIOR* \\ J orge Alberto OLIVEIRA* \\ António PRISTA*** \\ Go TANI* \\ J osé António Ribeiro MAIA ${ }^{* * * *}$
}

*Escola de Educação Física e Esporte, Universidade de São Paulo.

**Faculdade de Educação Física, Instituto Federal de Educação,

Ciência e Tecnologia do Sul de Minas Gerais. ***Faculdade de Ciências de Educação Física e Desporto, Universidade Pedagógica de Moçambique Moçambique.

***Faculdade de Desporto, Universidade do Porto - Portugal.

Estudos sobre coordenação motora grossa (CMG) de crianças focam na descrição dos valores normativos em razão da idade e sexo. Poucos analisam a dinâmica da mudança do desempenho de cada criança em relação ao seu grupo ao longo do tempo. 0 objetivo do presente estudo foi estimar a estabilidade das diferenças interindividuais na mudança intraindividual da CMG ao longo de 18 meses. Participaram do estudo 120 escolares de sete anos de idade da cidade de Muzambinho - MG. Foram realizadas quatro coletas com intervalos de seis meses. A CMG foi avaliada pelas provas do KTK. Os valores médios apresentaram incrementos em todas as provas, diferindo entre gênero apenas para o equilíbrio à retaguarda. Os resultados da correlação entre o desempenho inicial e a mudança ao longo do tempo indicaram uma forte heterogeneidade. Os resultados da estabilidade se mostraram fracos, os quais foram discutidos em relação a diferentes trajetórias do desenvolvimento da CMG.

UnITERMos: Estudo longitudinal; Diferenças interindividuais; Mudança intra-individual; "Tracking"; Crianças.

\section{Introdução}

O programa curricular de Educação Física de qualquer nível de ensino é o documento essencial na organização e estruturação do processo de ensino-aprendizagem de crianças e jovens em torno das diferentes manifestaçōes da cultura de movimento e das mais elevadas potencialidades do desenvolvimento da motricidade, da personalidade e da socialização. A partir do programa é de esperar que o professor construa unidades didáticometodológicas de assuntos nucleares da disciplina. A eficácia de sua implementação e alcance dos seus objetivos repousa, em boa medida, no conhecimento relativamente preciso e extenso das características dos alunos. Espera-se que o professor possua uma visão clara e profunda acerca da dinâmica do seu crescimento físico, da aprendizagem motora, do desenvolvimento motor, cognitivo e afetivo-social e respectivas interações para a elaboração dos grandes propósitos da sua ação educativa (LOPES, MAIA \& Mota, 2000; Tani, 1987; Tani, Gaya \& Marques, 2004; Tani, Manoel, Kokubun \& ProençA, 1988). É de sua responsabilidade o conhecimento preciso das competências e necessidades dos seus alunos.

No domínio do estudo do desenvolvimento motor estão disponíveis obras de diferentes perspectivas desenvolvimentistas (por exemplo, ConNolly, 1970; Gallahue \& Ozmun, 2005; Haywood \& Getchell, 2005; Malina, BOUChard \& BAR-OR, 2004; RoBERTON \& HalVERSON, 1984) que são da maior importância para o professor de educação física na condução do processo ensino-aprendizagem. Nestes livros-textos há muita informação acerca do comportamento médio de diferentes aspectos do desempenho motor de crianças e jovens em função da idade cronológica e sexo. 
Também há informação sobre algumas características da informação individual de aspectos do desenvolvimento motor, mais especificamente das capacidades físicas e de algumas das habilidades motoras básicas (ver, por exemplo, o Cap. 11 de Malina, Bouchard \& Bar-Or, 2004). No campo do crescimento somático esse tipo de informação já tem alguma tradição e as suas implicações são bem conhecidas (TANNER, 1990). Contudo, o mesmo não ocorre nas diferentes manifestaçôes da coordenação motora grossa de crianças e jovens ao longo de sua escolaridade. ScHILLING e KIPHARD (1974) têm descrições da coordenação motora grossa exclusivamente centradas nos valores médios; a mesma ênfase ocorre em investigações acerca da influência de variáveis ambientais e/ou organísmicas (CATENASSI, Marques, Bastos, Basso, Ronque \& Gerage, 2008; Graf, Koch, KretschmanNKandel, FalKowski, Christ, Coburger, Lehmacher, Bjarnason-Wehrens, Platen, Tokarski, Predel $\&$ Dordel, 2004; Lopes \& Maia, 1997; Lopes, Maia, Silva, Seabra \& Morais, 2003; Panagopoulou, Nakou, GiannaKoulia \& Serbezis, 2008; Valdivia, Cartagena, Sarria, Távara, Teixeira, da Silva \& Maia, 2008). Há um espaço substancial de exploração das características da informação individual que parece ter permanecido ausente das preocupaçôes dos pesquisadores da coordenação motora, fundamentalmente sobre a dinâmica da mudança de cada sujeito em relação ao seu grupo ao longo do tempo (MAIA, GARGANTA, Seabra, Lopes, Silva \& Meira Junior, 2007). Uma possível explicação para a ênfase apenas na descrição da mudança média do desenvolvimento da coordenação motora pode estar atrelada à pequena quantidade de estudos com informação longitudinal; na sua grande maioria, os estudos utilizam informação transversal. Isso impede qualquer questionamento sobre a mudança na coordenação motora de natureza individual.

A ausência desse tipo de informação e a sua análise e interpretação deixam em aberto questôes sobre as mudanças ocorridas na coordenação motora grossa ao longo do período escolar. Isto deixa sem resposta uma série de questôes, por exemplo: será que crianças com baixos desempenhos na coordenação motora logo no início da escolaridade teriam sempre os resultados mais baixos ao longo de todo o processo? E as crianças com melhores desempenhos no início, também teriam elas sempre desempenhos superiores por todo o processo educacional? Estudos empíricos sobre o "tracking" da coordenação motora grossa ao longo da idade escolar que evidenciem as diferenças interindividuais na mudança intra-individual são praticamente inexistentes (por exemplo, Coelho Deus, Bustamante, Lopes, Seabra, Silva \& Maia, 2008; Willimczik, 1980).
Para que se possa investigar essas questões é necessário definir o contexto e as ideias nucleares da concepção de "tracking". São três as essenciais: mudança, estabilidade e previsão. No âmbito do "tracking" a mudança é tida como um fenômeno de natureza intra-individual, uma propriedade intrínseca de cada unidade individual de observação (MAIA, BEUNEN, Lefevre \& Claessens, 1998). Com isso a pesquisa desenvolvimentista sobre a coordenação motora precisa enfocar a identificação direta da mudança ocorrida em cada indivíduo ao longo do tempo (NESSELROADE, 1991). Cabe também salientar que o foco deve ser na mudança verdadeira, de natureza irreversível e de longo prazo, distinguindo-a claramente da mudança de curto prazo, reversível, denominada de variabilidade intra-individual (NESSELROADE, 1991). Estudar a mudança da coordenação motora focada apenas nos valores normativos não garante entender o que acontece com os sujeitos ao longo do tempo, pois a variação entre os sujeitos pode não expressar apenas o ruído ou erro amostral - como pressupóe a análise normativa da mudança.

A segunda ideia fulcral é a de estabilidade. Bastante diversa e abrangente, podendo ser diferenciada segundo Mortimer, Finch e Kumka (1982) do seguinte modo: a) estabilidade estrutural - referindose ao grau de continuidade do fenômeno sobre investigação; b) estabilidade normativa - o foco reside na estabilidade das diferenças interindividuais e nas mudanças intra-individuais; c) estabilidade de nível referindo-se à persistência da magnitude da mudança ao longo do tempo num traço ou característica; e d) estabilidade ipsativa - referindo-se à consistência e à mudança intra-individual na organização dos traços ou atributos ao longo do tempo. No presente estudo, as questões de investigação que foram apresentadas no inicio do texto podem ser respondidas com base na estabilidade normativa, pois o foco da estabilidade do presente estudo reside na análise da manutenção da posição relativa do sujeito na distribuição dos valores do seu grupo ao longo do tempo.

É somente com base na estabilidade normativa de um determinado comportamento que se torna possível discutir, com alguma segurança, sobre a predição do comportamento no futuro. Com a estabilidade normativa é possível efetuar previsões acerca dos níveis de coordenação motora e assim adequar tanto os objetivos das opçôes didático-metodológicas dos professores quanto as expectativas do desempenho dos seus alunos. Por outro lado, a baixa estabilidade em certas características da coordenação motora impossibilita a precisão da previsão. Contudo, carrega 
consigo a possibilidade de ser interpretada como tendo um alto potencial para a mudança e, portanto, um momento importante para a intervenção pedagógica.

A ideia de mudança, estabilidade e previsão são os elementos nucleares da concepção de "tracking" (para uma revisão, MAIA et al., 2007). Embora exista um consenso relativo à noção genérica de que o "tracking" traduz a tendência de um sujeito ou grupo de sujeitos permanecerem num dado curso de mudança, isto é, a manutenção da posição relativa de um sujeito dentro da distribuição ao longo do tempo, dois aspectos devem ser ressaltados: a) a diversidade de sua operacionalização estatística, por exemplo, mediante autocorrelação; $\kappa$ de Cohen; Gama $(\gamma)$ de Foulkes e Davis; b) ausência de uma definição operacional unânime acerca do que se entende por posição relativa (MAIA et al., 2007).

A utilização das diferentes operacionalizações estatísticas do "tracking" está ligada diretamente às questôes de estudo. No entanto, entende-se que a

\section{Método}

\section{Amostra}

A amostra é proveniente do estudo longitudinalmisto realizado com crianças da cidade de Muzambinho. Genericamente, esta pesquisa foi delineada para avaliar e interpretar diferentes aspectos do crescimento físico, desenvolvimento motor e aptidão física de crianças e adolescentes de seis a 14 anos de idade, matriculados na rede pública de ensino. Nesse sentido, foi concebida uma estrutura amostral dividida em cinco coortes. No presente estudo serão analisados dados de 120 crianças (56\% meninos e $44 \%$ meninas) de sete anos que foram analisadas semestralmente até aos 8,5 anos de idade, tendo assim, quatro momentos de avaliação.

\section{Bateria de testes}

Para avaliar a coordenação motora grossa foi utilizada a bateria de testes KTK (Körperkoordinationstest für Kinder) de SCHILLING e KIPHARD (1974), constituída por quatro testes: equilíbrio em marcha à ré (ER), saltos monopedais (SM), saltos laterais (SL) e transferência lateral sobre plataformas (TL). O desempenho da criança refere-se aos aspectos quantitativos, sendo registrado o número de passos na marcha à ré, a altura mais elevada dos saltos, o número de saltos laterais, e o número de transposições realizadas. Face à inexistência combinação de diferentes estatísticas permite um aprofundamento no entendimento das informações sobre a estabilidade na mudança intra-individual do desempenho da coordenação motora ao longo do tempo, permitindo leituras mais extensas e diversificadas do fenômeno.

O presente estudo, realizado com crianças de sete aos 8,5 anos de idade, tem os seguintes objetivos: 1) explicitar ideias e técnicas estatísticas para análise de informação longitudinal da coordenação motora grossa para além da análise dos valores médios; 2) apresentar a diversidade das mudanças intra-individuais no desempenho dos quatro testes coordenativos considerando exclusivamente a diferença do quarto para o primeiro momento de avaliação e a sua associação com os valores iniciais de desempenho; 3 ) estimar a estabilidade do desempenho da coordenação motora grossa e discutir as suas implicaçôes na área pedagógica da educação física.

de estudos de natureza transcultural no que se refere a validade de constructo da ideia de Coeficiente Motor e dos valores de corte que se utiliza para classificar as crianças em categorias distintas, optou-se pelo estudo e análise individual de cada teste. A bateria KTK que mede aspectos da coordenação motora, por sua natureza e origem, se baseia em constructos; porém, ao que consta, no Brasil e atualmente, não se tem evidências empíricas de que ela mede a coordenação motora a partir de um único fator. Ela foi elaborada nos meados da década de 70 do século passado para crianças alemãs. Assim, a sua validade na realidade de Muzambinho não pode ser "a priori" afirmada, tornando-se mais prudente não assumir sua estrutura e valores de classificação.

\section{Procedimentos estatísticos}

Todos os dados coletados foram anotados numa ficha individual, sendo devidamente digitados em dupla entrada. A transformação do desempenho de cada tentativa em pontos para cada teste foi realizada de acordo com o sistema de pontuação da própria bateria do KTK. Essa opção ainda que também não possua um equivalente para a população brasileira, não traz consigo os problemas de análise e interpretação decorrentes do coeficiente motor.

A análise exploratória de dados constou, dentre outros aspectos, da identificação de "outliers" mediante 
inspeção da representação gráfica do diagrama de extremos e quartis. A análise da normalidade das distribuições foi realizada mediante o teste de ShapiroWilk. Para o cálculo da estatística descritiva básica (média e desvio padrão e suas respectivas representaçōes gráficas) e para testar o efeito dos fatores tempo e sexo por meio da Anova mista [efeito temporal e efeito fixo (sexo)]. As análises foram realizadas no SPSS v.16.

Para a análise de um dos aspectos da dinâmica das mudanças intra-individuais do quarto para o primeiro momento foram calculadas as diferenças intra-individuais dos desempenhos e efetuadas a sua representação gráfica. Para investigar a associação entre o desempenho inicial e a mudança da primeira para a quarta avaliação foi utilizada a correlação de Pearson e sua representação gráfica foi realizada no "software" Systat v.10. Essas mesmas análises foram novamente realizadas considerando a divisão da amostra em dois subgrupos: um formado pelas crianças com desempenho acima do valor mediano e outro abaixo do mesmo valor. Para efeito ilustrativo também foi calculado o valor da diferença da quarta para a primeira avaliação em termos percentuais para cada um dos testes: delta $=[($ avaliação4-avaliação 1$) /$ avaliação1] ${ }^{*} 100$.

A análise de "tracking" foi efetuada no programa estatístico Longitudinal Data Analysis (LDA), construído especialmente para lidar com alguns dos problemas decorrentes da análise de informação longitudinal. O "tracking" foi descrito por duas estatísticas conceitualmente distintas, mas complementares: o Gama $(\gamma)$ de Foulkes e Davis e

\section{Resultados}

Os resultados serão apresentados em termos descritivos/normativos para meninos e meninas em cada um dos testes do KTK nas quatro avaliaçōes. Em seguida, são apresentados os aspectos da dinâmica das mudanças intra-individuais nos testes de coordenação, e finalmente os valores estatísticos de "tracking" calculados pelo $\kappa$ de Cohen e $\gamma$ de Foulkes e Davis, com suas ilustrações gráficas. o Kappa de Cohen ( $\kappa)$. O $\gamma$ de Foulkes e Davis é uma estatística descritiva do "tracking" que calcula a probabilidade de duas trajetórias aleatórias de desempenho serem paralelas (FoulKes \& DAVIS, 1981). Valores de $\gamma$ abaixo de 0,5 indicam ausência de "tracking"; acima de 0,5 indicam a existência de algum "tracking" e quando igual a 1, o "tracking" é perfeito. Essa última condição ocorre quando for mantida a posição relativa dos indivíduos no seio da distribuição de trajetórias interindividuais. No presente artigo optou-se pela análise do $\kappa$ a partir da trajetória de cada criança. A estatística do $\kappa$ de Cohen estipula a existência de "tracking" se os sujeitos tenderem a permanecer no mesmo tercil ("track" ou canal) da distribuição e analisa quantas vezes o desempenho do sujeito está no seu tercil ao longo do tempo. É uma estatística descritiva não paramétrica, e assim não exige qualquer tipo de estruturação na distribuição dos dados. Baseado nas sugestôes de LANDIS e KOCH (1977) considera-se a seguinte interpretação para os valores: $\kappa>0,75 \kappa$ estabilidade excelente; $0,40<\kappa<$ $0,75=$ estabilidade moderada; $\kappa<0,40=$ estabilidade fraca. O estudo do "tracking" com base no $\kappa$ de Cohen é de máxima importância pela circunstância de se pensar que os desempenhos temporais das crianças possam pertencer a diferentes canais, que refletem sub-grupos de níveis distintos de prontidão coordenativa. Essa noção pode também veicular distintas possibilidades de sensibilidade de resposta a estímulos das aulas em relação ao desenvolvimento coordenativo. No caso referente só foi considerada, em termos exploratórios, a presença de três canais.

\section{Análise descritiva normativa}

Há um aumento gradual no desempenho médio de meninos e meninas em todos os testes ao longo das quatro avaliações, com exceção do ER, que apresenta oscilações entre as avaliaçóes tanto para os meninos quanto para as meninas (TABELA 1 e FIGURA 1). Os resultados da Anova mista indicaram efeito significativo apenas para o tempo em ambos os sexos nos testes de SL, SM e TL e interação entre sexo e tempo para o teste de ER, com valores maiores para os meninos apenas na última avaliação. 
TABELA 1 - Valores médios ( \pm ) desvios-padrão, valores mínimos e máximos do desempenho dos quatro testes da bateria KTK em cada avaliação para meninos e meninas.

\begin{tabular}{|c|c|c|c|c|c|c|c|c|c|}
\hline & \multirow[b]{2}{*}{ Teste } & \multicolumn{2}{|c|}{$1^{\text {a }}$ avaliação } & \multicolumn{2}{|c|}{$2^{a}$ avaliação } & \multicolumn{2}{|c|}{$3^{a}$ avaliação } & \multicolumn{2}{|c|}{$4^{a}$ avaliação } \\
\hline & & $M \pm d p$ & Min-Max & $M \pm d p$ & Min-Max & $M \pm d p$ & Min-Max & $M \pm d p$ & Min-Max \\
\hline \multirow{4}{*}{$\begin{array}{l}\stackrel{0}{\Xi} \\
\stackrel{\Xi}{\Xi} \\
\sum\end{array}$} & ER & $42,15 \pm 13,36$ & $13-66$ & $40,30 \pm 5,93$ & $28-51$ & $44,03 \pm 11,26$ & $25-65$ & $46,38 \pm 10,51$ & $25-64$ \\
\hline & SL & $30,11 \pm 6,85$ & $20-50$ & $34,46 \pm 7,78$ & $21-50$ & $41,15 \pm 8,41$ & $25-59$ & $44,76 \pm 9,41$ & $21-63$ \\
\hline & SM & $28,72 \pm 14,83$ & $2-59$ & $34,06 \pm 13,90$ & $9-63$ & $47,58 \pm 13,35$ & $18-72$ & $52,06 \pm 14,58$ & $26-72$ \\
\hline & $\mathrm{TL}$ & $29,31 \pm 4,51$ & $20-40$ & $33,93 \pm 5,05$ & $26-44$ & $38,55 \pm 5,62$ & $28-48$ & $42,41 \pm 5,35$ & $32-54$ \\
\hline \multirow{4}{*}{$\frac{\stackrel{\mathscr{J}}{\Xi}}{\stackrel{\Xi}{\Xi}}$} & ER & $39,21 \pm 9,37$ & $20-57$ & $43,21 \pm 10,87$ & $18-62$ & $43,78 \pm 11,62$ & $22-66$ & $40,89 \pm 12,01$ & $18-61$ \\
\hline & SL & $35,32 \pm 6,58$ & $24-49$ & $41,92 \pm 10,74$ & $23-61$ & $46,72 \pm 8,86$ & $29-62$ & $49,64 \pm 8,90$ & $32-63$ \\
\hline & SM & $31,82 \pm 11,39$ & $7-54$ & $35,57 \pm 13,80$ & $12-64$ & $48,07 \pm 14,76$ & $20-71$ & $50,67 \pm 16,68$ & $12-72$ \\
\hline & $\mathrm{TL}$ & $26,62 \pm 5,01$ & $16-34$ & $31,65 \pm 5,29$ & $20-40$ & $35,31 \pm 5,68$ & $24-44$ & $38,68 \pm 4,54$ & $30-46$ \\
\hline
\end{tabular}

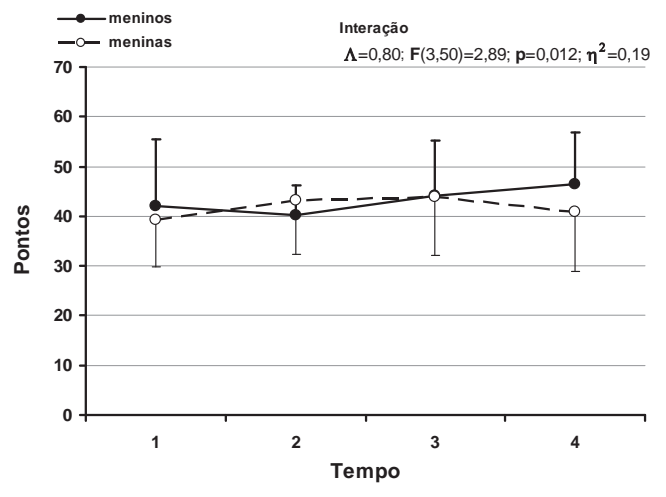

(ER)

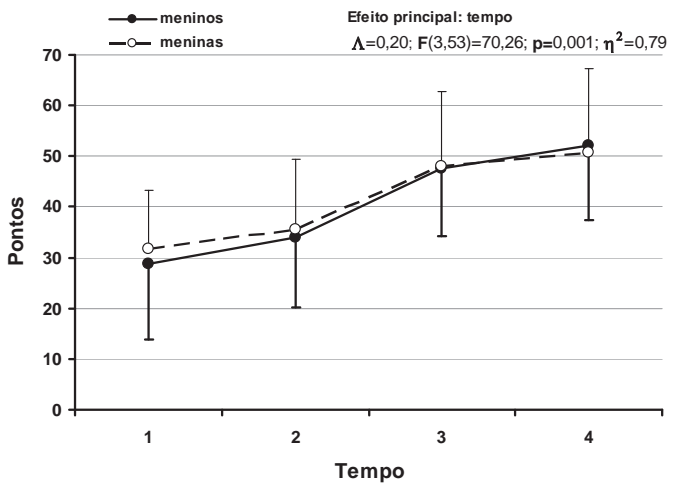

$(\mathrm{SM})$

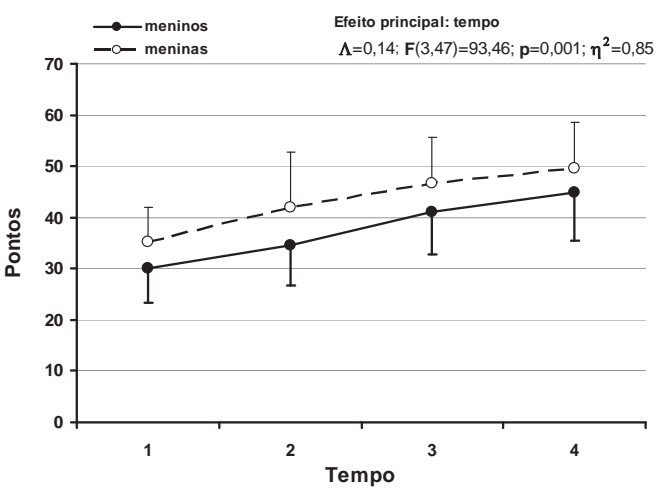

$(\mathrm{SL})$

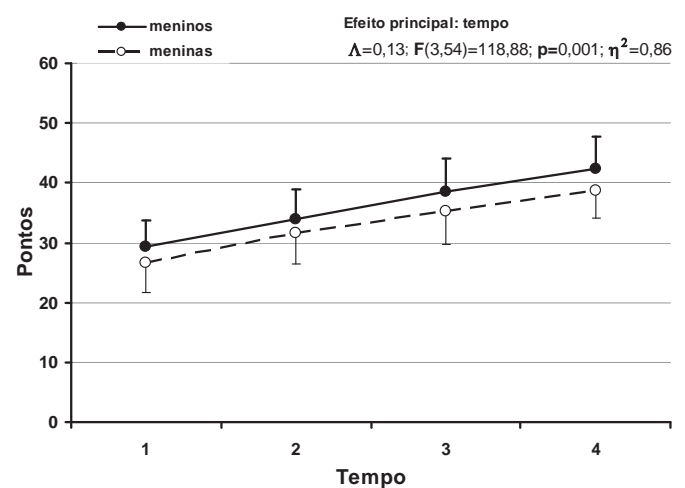

(TL)

FIGURA 1 - Valores médios e desvios padrão do desempenho dos quatro testes do KTK ao longo das quatro avaliações para meninos e meninas. 


\section{Análise da dinâmica das mudanças do quarto para o primeiro momento ativa}

Os valores dos desempenhos médios e respectivos desvios-padrão apresentados acima nada informam sobre as diferenças entre as crianças na magnitude da mudança intra-individual. Diante disso, opta-se pelas representações gráficas das FIGURAS 2 e 3. É notória uma grande diversidade na magnitude da mudança entre as crianças (FIGURA 2), evidenciando uma clara heterogeneidade na sua sensibilidade de resposta ao processo da sua ontogênese coordenativa grossa. Por exemplo, no teste ER há crianças que têm redução no seu desempenho coordenativo, há crianças que mostram silêncio de resposta e há ainda crianças que evidenciam ganhos díspares, não obstante a expectativa que a da ação educativa implicasse ganhos para todas as crianças. Os testes SL, TL e SM apresentam padróes de mudanças intra-individual distintos na sua magnitude.

Um padrão semelhante nas diferenças entre crianças é observado quando a amostra é dividida

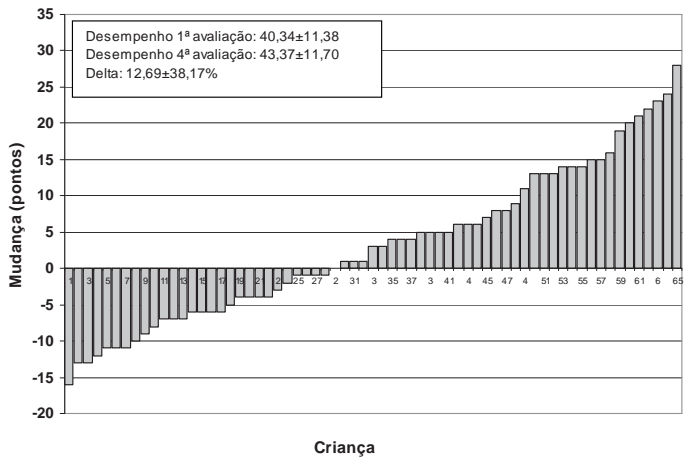

(ER)

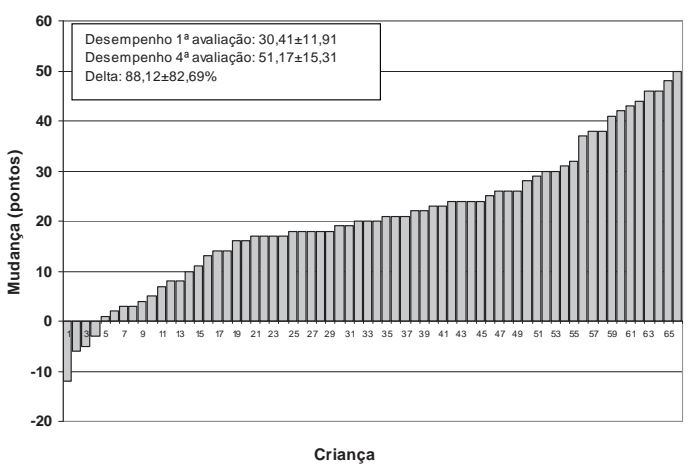

$(\mathrm{SM})$ em dois subgrupos (acima e abaixo do valor da mediana). No entanto, parece haver uma mudança de maior magnitude no grupo que tem um desempenho inicial mais baixo, observado na FIGURA 3 à esquerda, e pelo valor do delta percentual.

Uma questão importante desta pesquisa com implicações pedagógicas evidentes é saber se a mudança ocorrida na coordenação grossa dos sete para os 8,5 anos de idade depende da prontidão coordenativa das crianças aos sete anos de idade. Essa questão foi abordada em duas etapas sequenciais: considerando a totalidade das crianças e a sua divisão em relação ao valor mediano do desempenho aos sete anos de idade (FIGURA 4). Com exceção do SL, os valores da correlação ( $r$ negativo) mostram que quanto maior o desempenho aos sete anos de idade menor é o valor da mudança. No entanto, para o SM, a correlação é a mais fraca (como se pode observar à esquerda da FIGURA 4). Cabe lembrar que esse padrão diminui quando se considera a divisão do grupo pela mediana, persistindo apenas para o ER.

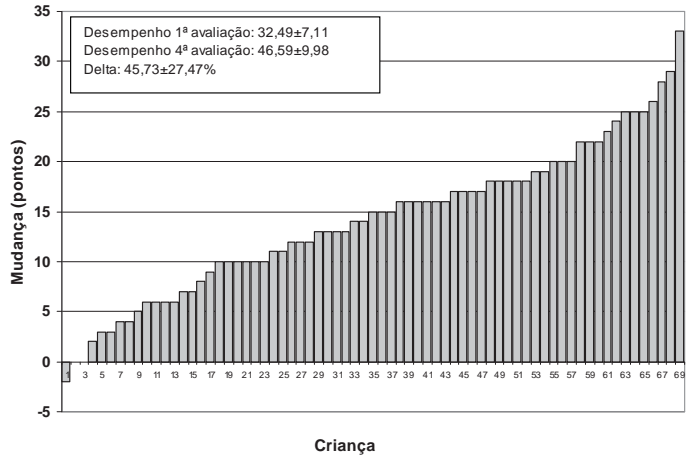

(SL)

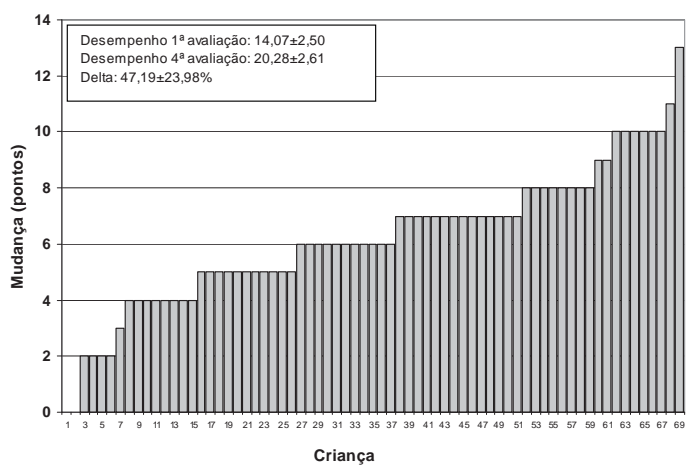

(TL)

FIGURA 2 - Heterogeneidade nas mudanças do desempenho intra-individual da primeira para a quarta avaliação nos testes de equilíbrio em marcha à ré (ER), saltos laterais (SL), saltos monopedais (SM) e transposição lateral (TL). 

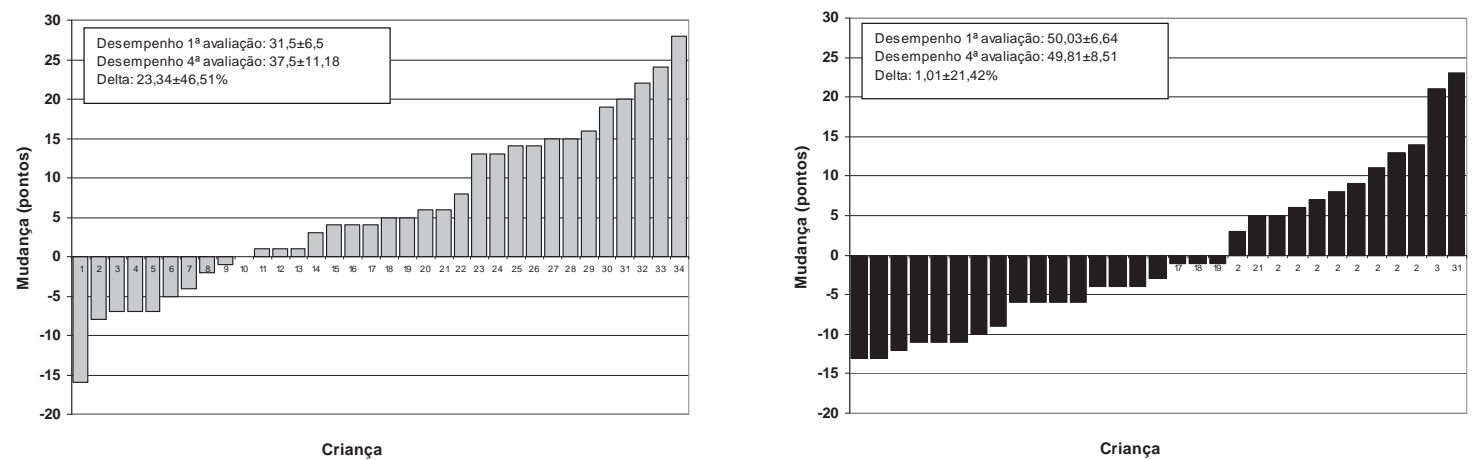

(ER)
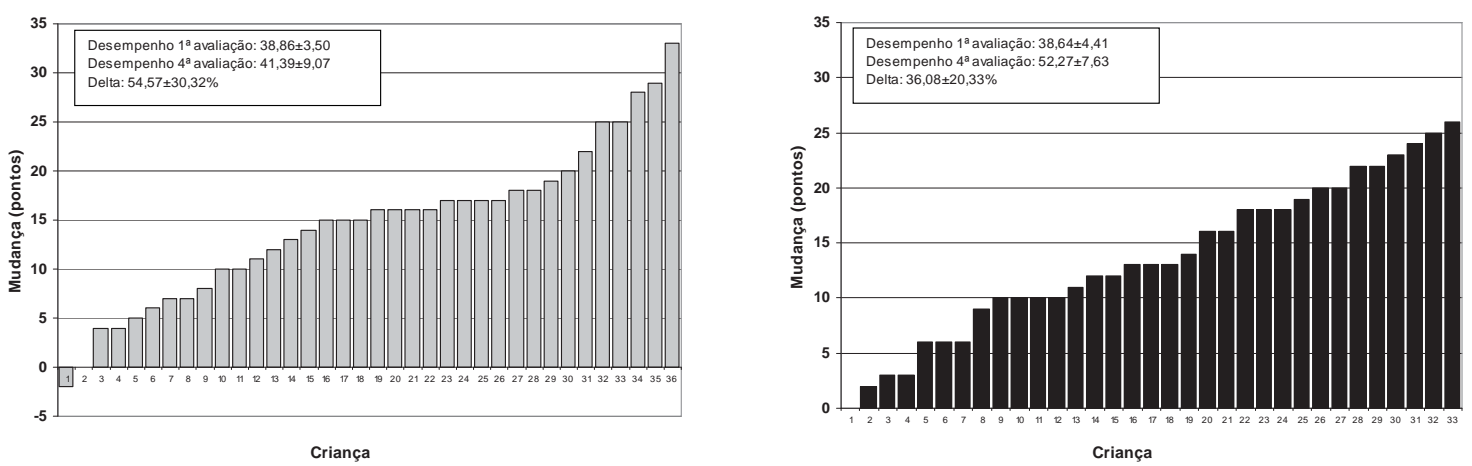

(SL)
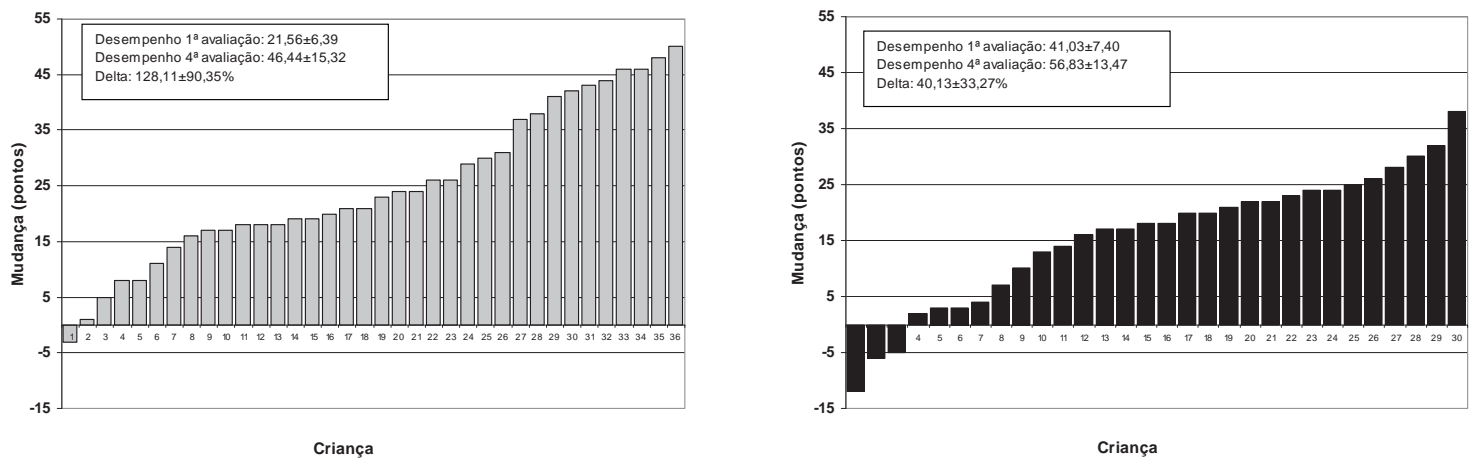

$(\mathrm{SM})$
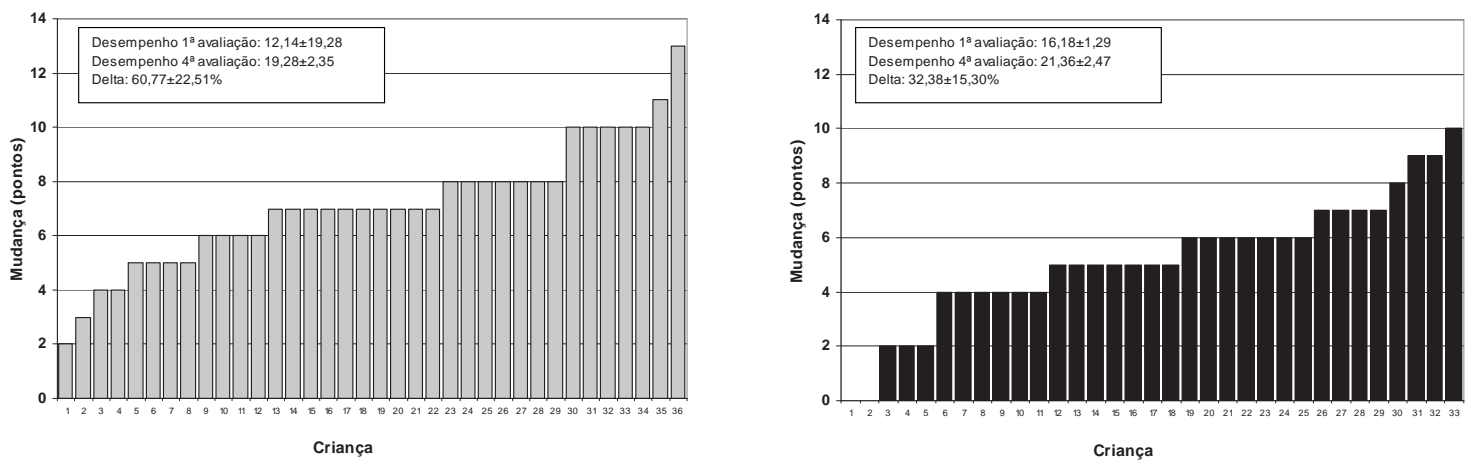

(TL)

FIGURA 3 - Heterogeneidade nas mudanças do desempenho intra-individual da primeira para a quarta avaliação nos testes de equilíbrio em marchaàré(ER), saltos laterais (SL), saltos monopedais (SM) etransposição lateral (TL) para as crianças classificadas abaixo (à esquerda) e acima (à direita) da mediana. 

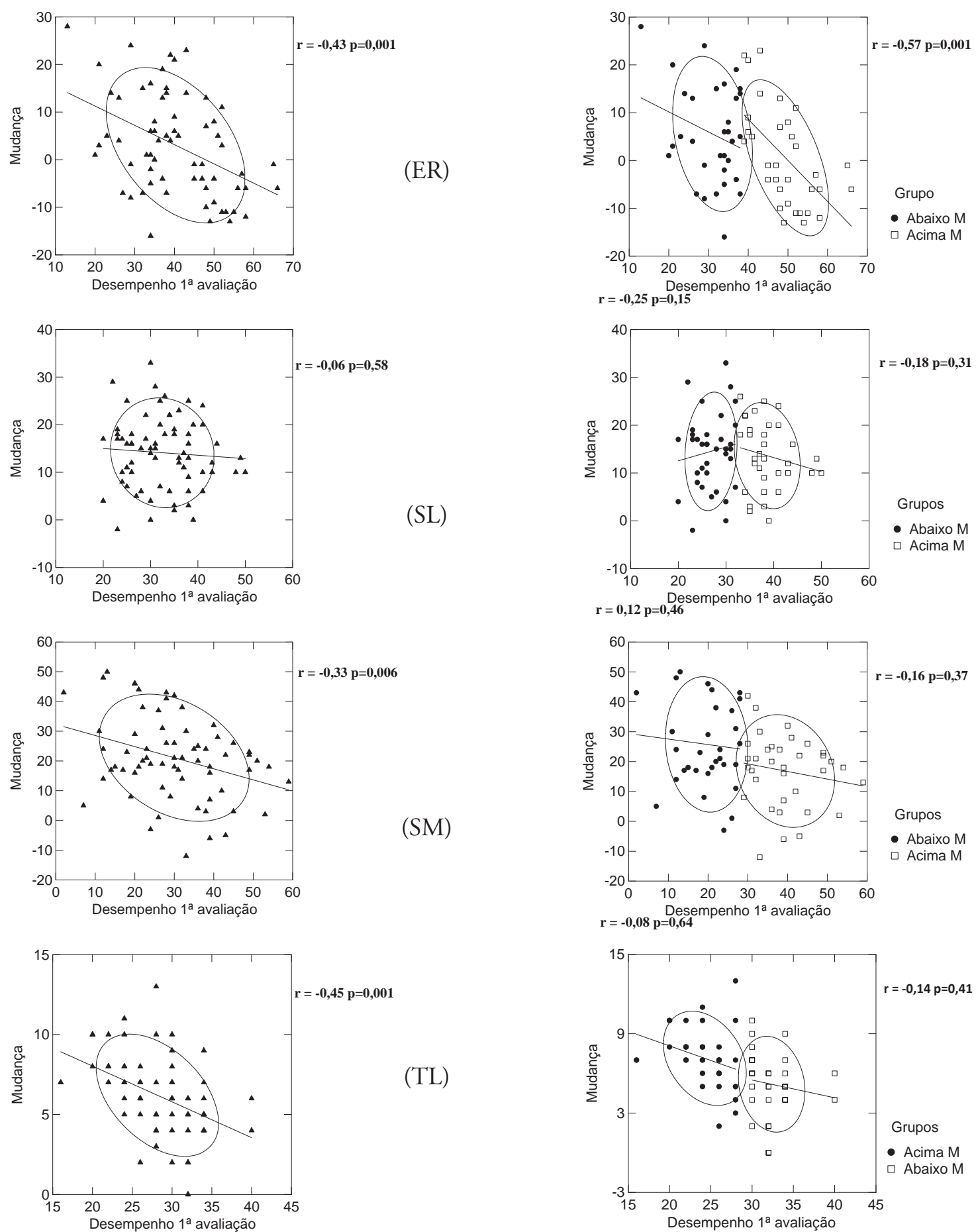

FIGURA 4 - Diagrama de dispersão dos valores do desempenho coordenativo na primeira avaliação e dos valores da mudança da primeira para a quarta avaliação e respectivos valores do r para a amostra total (à esquerda) e para os subgrupos acima e abaixo da mediana (à direita).

Análise do tracking: $\gamma$ de Foulkes e Davis

Os valores do $\gamma$ de Foulkes e Davis para cada um dos testes para meninos e meninas (TABELA 2) indicam não haver estabilidade no desempenho ao longo do tempo para nenhum dos testes. A inexistência de "tracking" nas trajetórias individuais reflete uma elevada quantidade de cruzamentos de perfis das mudanças entre crianças ao longo das avaliaçôes. 
TABELA 2 - Valores de $\gamma \pm$ erro padrão e intervalo de confiança a 95\% nos diferentes testes do KTK para meninos e meninas.

\begin{tabular}{|c|c|c|c|}
\hline & & $\gamma \pm$ ep & IC $95 \%$ \\
\hline \multirow{4}{*}{ 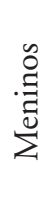 } & ER & $0,08 \pm 0,02$ & $0,06-0,10$ \\
\hline & SL & $0,09 \pm 0,02$ & $0,07-0,11$ \\
\hline & SM & $0,12 \pm 0,02$ & $0,09-0,14$ \\
\hline & $\mathrm{TL}$ & $0,12 \pm 0,02$ & $0,09-0,14$ \\
\hline \multirow{4}{*}{ 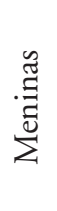 } & ER & $0,17 \pm 0,02$ & $0,13-0,21$ \\
\hline & SL & $0,13 \pm 0,02$ & $0,10-0,16$ \\
\hline & SM & $0,18 \pm 0,03$ & $0,14-0,22$ \\
\hline & $\mathrm{TL}$ & $0,19 \pm 0,02$ & $0,14-0,23$ \\
\hline
\end{tabular}

\section{Análise do "tracking”: $\kappa$ de Cohen}

Os resultados da estabilidade em diferentes canais com base no $\kappa$ de Cohen estão na TABELA 3. Para nenhum dos testes o valor do $\kappa$ indica a permanência dos sujeitos em apenas um canal, ou seja, os baixos valores do $\kappa$ indicam que a trajetória individual oscila entre os três canais (para efeito ilustrativo os valores dos canais e trajetórias individuais apenas das meninas são apresentados na FIGURA 5). Apenas os valores para SM e TL no primeiro canal indicam uma estabilidade moderada para as meninas. O teste TL mostra diferenças significativas nos valores de $\kappa$ entre meninos e meninas.

TABELA 3 - Valoresdo $\kappa \pm$ erropadrãoparaostrêscanaiseporcanal $(\kappa 1, \kappa 2$ e $\kappa 3)$ nosquatrotestes do KTKparameninos emeninas, bem como valones de $\kappa$ e $\chi^{2}$ para a comparação da estabilidade do desempenho entre sexos.

\begin{tabular}{|c|c|c|c|c|c|c|c|c|c|c|c|c|}
\hline & \multicolumn{4}{|c|}{ Meninos } & \multicolumn{4}{|c|}{ Meninos } & \multirow{2}{*}{\multicolumn{4}{|c|}{$\begin{array}{c}\text { Comparação } \\
\text { meninos - meninas } \\
\text { Geral }\end{array}$}} \\
\hline & Geral & & Canais & & Geral & & Canais & & & & & \\
\hline & $\kappa \pm$ ep & $\kappa 1$ & $\kappa 2$ & $\kappa 3$ & $\kappa \pm$ ep & $\kappa 1$ & $\kappa 2$ & $\kappa 3$ & $\kappa$ & Ic $95 \%$ & $\chi^{2}$ & $\mathrm{p}$ \\
\hline ER & $0,13 \pm 0,02$ & 0,36 & 0,09 & 0,02 & $0,19 \pm 0,02$ & 0,41 & 0,11 & 0,26 & 0,22 & $0,18-0,25$ & 2,67 & 0,10 \\
\hline SL & $0,12 \pm 0,02$ & 0,29 & 0,13 & 0,10 & $0,15 \pm 0,03$ & 0,29 & 0,09 & 0,34 & 0,20 & $0,16-0,23$ & 0,23 & 0,63 \\
\hline SM & $0,15 \pm 0,02$ & 0,34 & 0,11 & 0,19 & $0,20 \pm 0,03$ & 0,48 & 0,08 & 0,27 & 0,24 & $0,20-0,28$ & 2,45 & 0,11 \\
\hline $\mathrm{TL}$ & $0,13 \pm 0,02$ & 0,26 & 0,12 & 0,14 & $0,21 \pm 0,03$ & 0,48 & 0,18 & 0,18 & 0,22 & $0,18-0,25$ & 8,80 & 0,02 \\
\hline
\end{tabular}

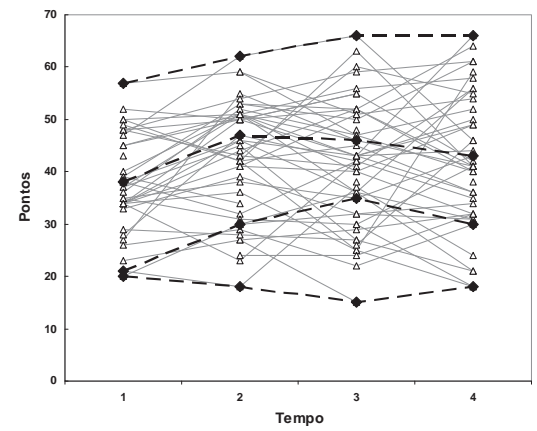

(ER)

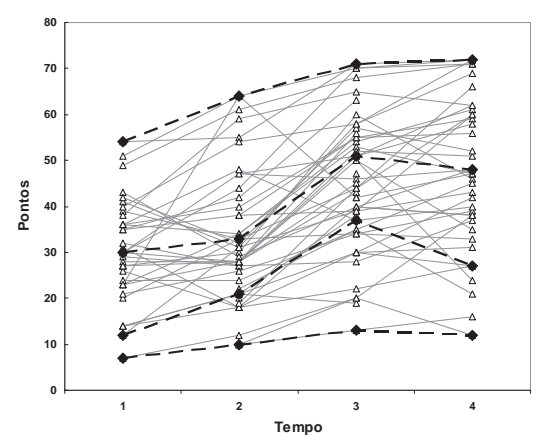

$(\mathrm{SM})$

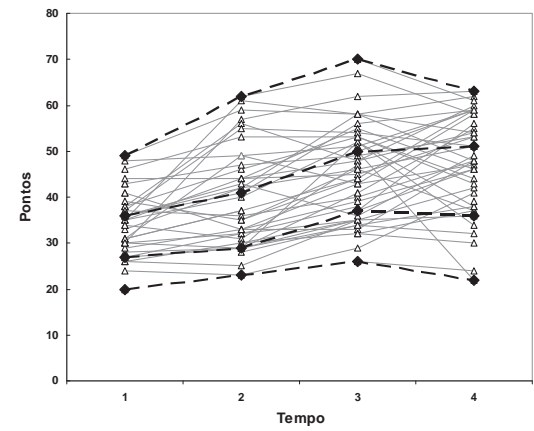

(SL)

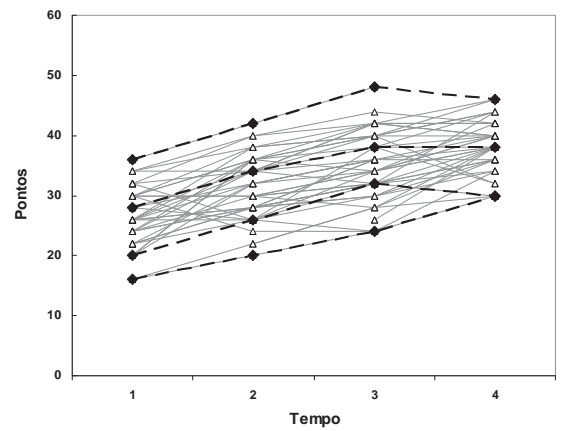

(TL)
As linhas tracejadas e pontos cheios indicam os diferentes canais.

FIGURA 5 - Trajetóniasintra-individuaisediferençasinterindividual dasmeninasaolongo das quatroavaliagõesnostestes de equilíbrio em marcha à ré(ER), saltos laterais (SL), saltos monopedais (SM) etransposição lateral (TL). 


\section{Discussão}

O estudo do desenvolvimento da coordenação motora grossa, qualquer que seja o seu contexto, padece de um problema sério: a falta de uma visão integradora entre o modelo teórico da mudança, o delineamento temporal fundamentado e o modelo estatístico adequado para ajudar a descrever e interpretar as mudanças (Collins, 2006). Por esses motivos que esta pesquisa, essencialmente exploratória e empírica, tenha centrado a sua atenção na interpretação multifacetada da informação longitudinal da coordenação motora grossa de crianças dos sete aos 8,5 anos de idade. Além da descrição dos valores normativos de cada teste, foi analisada a heterogeneidade das mudanças intra-individuais ocorridas no período de um ano e meio e estimada a estabilidade das diferenças interindividuais na mudança intra-individual. Entende-se que pesquisas dessa natureza ampliem o conhecimento sobre a mudança da coordenação motora grossa ao longo da idade, cuja relevância é evidente no contexto escolar. Para além da informação do comportamento das médias esse conhecimento pode apresentar-se mais próximo dos aspectos pedagógicos no processo ensino-aprendizagem, provendo uma caracterização sobre a estabilidade do percurso de mudança de cada criança, o que possibilita refletir sobre o quão se pode prever do desenvolvimento da coordenação na faixa etária do presente estudo. O conhecimento sobre a previsibilidade do curso desenvolvimentista da coordenação motora individual e do grupo tem implicações na definição de objetivos do processo ensino-aprendizagem.

\section{Valores normativos}

Os resultados das análises normativas indicaram que a flecha do tempo cronológico é um forte preditor da mudança no desempenho para todos os testes. Houve tendência de incrementos médios ao longo do tempo para três testes (SL, SM e TL) e interação entre tempo e sexo para o teste de equilíbrio em marcha à ré (FIGURA 1). O incremento das médias ao longo do tempo também é reportado em estudos com crianças alemãs (SCHILLING \& KipHARD, 1976), portuguesas (LOPEs et al., 2003) e peruanas (VAlDIVIA et al., 2008), a apesar desses estudos indicarem efeito significativo para a interação entre sexo e tempo, implicando num efeito moderador do sexo, ampliando os valores dos meninos e "reduzindo" os valores das meninas. Nem sempre é claro se isso se deve a um aproveitamento diferenciado das oportunidades de experiências motoras, que pode refletir aspectos diversificados de natureza sociocultural relativa aos estereótipos esperados das experiências motoras lúdicas formais e não formais dos dois sexos. No presente trabalho a diferenciação entre sexos no desempenho não ocorreu. Pode daqui especular-se que meninos e meninas de Muzambinho foram, aparentemente, expostos a condiçōes de solicitação da sua motricidade que possibilitaram experiência suficiente para se desenvolverem de forma semelhante na sua coordenação motora. Ao comparar os resultados do estudo inicial de SCHILLING e KIPHARD (1976), não obstante alguma discrepância etária, nota-se que os valores médios são descritivamente superiores às de Muzambinho (por exemplo, no caso da TL, a diferença no desempenho médio entre as duas populaçōes é de aproximadamente $35 \%$ a favor das crianças alemãs). A exceção é verificada para o desempenho dos meninos nos testes de equilíbrio em marcha à ré aos sete anos e saltos monopedais aos oito anos e para as meninas também no salto monopedal aos oito anos de idade. Com relação às crianças portuguesas (Lopes et al., 2003) e peruanas (VAlDivia et al., 2008) o mesmo não ocorre, pois descritivamente os valores médios são mais próximos e não indicam uma superioridade de alguma das populaçôes.

\section{Dinâmica da mudança intra-individual}

É mais que sabido que a análise do comportamento da média em função do tempo não detecta toda a potencialidade da informação longitudinal (COELHO Deus et al., 2008; WILLIMCZIK, 1980). Quando o foco reside nas mudanças intra-individuais no período de um ano e meio pode-se observar que a sua magnitude não é semelhante para todas as crianças (FIGURA 2). Os resultados indicam uma clara heterogeneidade na sensibilidade de resposta das crianças ao processo da sua ontogênese coordenativa grossa. Análises e resultados dessa natureza não foram encontrados na literatura, mas é possível apresentar alguns aspectos para a discussão desses resultados (a relevância deste tipo de análise é bem salientada por BOUCHARD e RANKINEN, 2001 no contexto de indicadores fisiológicos).

É inquestionável que alteraçōes no crescimento somático induzem respostas diversas no desempenho motor (por exemplo, MALINA, BOUCHARD \& BAROr, 2004). O mesmo acontece na coordenação motora grossa, sobretudo quando se considera o 
efeito negativo do IMC a partir da informação transversal (Graf et al., 2004; VALDivia et al., 2008) ou longitudinal (Coelho Deus et al., 2008; D'Hondt, Deforche, Gentier, De Bourdeaudhuij, Vaeyens, Philippaerts \& Lenoir, 2012; Graf, Koch, FALKOWSKI, Jouck, Christ, STAUENMAIER, BJARNASONWehrens, Tokarski, Dordel \& Predel, 2005). Esses efeitos podem ajudar a interpretar a variação que ocorre no desempenho em cada valor discreto de idade, como também nas diferenças interindividuais nos ganhos coordenativos. Adicionalmente, as diferenças interindividuais na magnitude e sentido das mudanças podem estar associadas aos aspectos do planejamento pedagógico do professor de Educação Física. É um fato muito evidente que uma parte substancial do desenvolvimento motor da criança se processe no contexto escolar cabendo, sobretudo, à disciplina de Educação Física uma parcela importante da sua promoção. É nesse sentido que os Parâmetros Curriculares Nacionais enfatizam diferentes manifestaçôes da cultura de movimento (jogo, dança, ginástica - LDB, 2004). Contudo, nas séries iniciais é recomendado mais a diversificação desses conteúdos do que a sua especialização, o que acarretaria em algum incremento na coordenação motora para todas as crianças após um período de um ano e meio. No entanto, a forte heterogeneidade nos ganhos pode sugerir que, não obstante o cuidado e planejamento pedagógico do professor, a ausência de um conhecimento mais preciso sobre os níveis de prontidão coordenativa da criança induziria resultados distintos daqueles prescritos nos objetivos do professor. Essa indicação surge dos resultados salientados na FIGURA 3.

Os resultados de correlação entre a mudança no período de um ano e meio e o desempenho aos sete anos de idade para os testes de ER e TL indicam que há uma associação negativa entre o desempenho inicial e a mudança (ver os valores de correlação na FIGURA 4 à esquerda). Com isso, pode-se inferir que as maiores mudanças ocorreram para as crianças com menores desempenhos iniciais. Apesar de serem valores de correlação baixos, pois nenhuma correlação ultrapassou um $r=-0,45$, entende-se que esses representam em algum grau uma associação importante, pois o processo de mudança no domínio motor é multidimensional, e assim, está associado a um quadro diversificado de fatores e restrições (CONNOLLY, 1970; Gallahue \& OzMun, 2005; HayWOOd \& GeTChell, 2005; Malina, Bouchard \& Bar-Or, 2004; RoBerton \& Halverson, 1984). Como esse estudo é observacional e descritivo, não é possível afirmar sobre essa hipótese, mas a elaboração de diferentes hipóteses é uma possibilidade que enriquece a discussão da mudança no desempenho da coordenação motora ao longo da infância. Com isso, optou-se por apresentar duas formas: a primeira centrada na questão de haver, eventualmente, um menor espaço para a mudança para crianças com maior desempenho inicial, pois já estariam próximas dos seus valores máximos - a hipótese é que haveria um efeito teto no desenvolvimento da coordenação motora; a segunda centra-se na ideia de haver alguma preocupação de adequar as atividades de aula às crianças com menores desempenhos iniciais. Essa continua a ser um grande desafio na organização didático-metodológica das aulas de modo que os seus conteúdos cheguem a todos na medida da necessidade e nível de prontidão.

Com base nos valores da correlação entre a mudança e o desempenho inicial para os subgrupos acima e abaixo da mediana é possível notar que os valores significativos desaparecem. Aparentemente houve um efeito de dois subgrupos de dados não correlacionados quando em conjunto apresentam uma correlação significativa. A exceção foi o equilíbrio em marcha à ré, que mantém valores negativos de correlação para ambos os subgrupos, indicando que as crianças que apresentaram maiores mudanças eram as que tinham valores iniciais mais baixos, independente de apresentarem maiores ou menores níveis de prontidão motora inicial. Com base nesses resultados pode-se levantar a hipótese interpretativa de que o professor, ao estabelecer seus objetivos e estratégias didático-metodológicas, pode não ter levado em consideração os distintos níveis de prontidão das crianças no que se refere à coordenação motora.

É importante lembrar que essas interpretações são elaboradas a partir do princípio de que a criança está sempre pronta para algum tipo de experiência (BRUNNER, 1965), e assim possa aprender qualquer assunto, mas a sua capacidade para aprender não é apenas atribuída ao seu nível de desenvolvimento no início da aprendizagem da tarefa. É atribuída à combinação entre a proficiência prévia e uma série de experiências apropriadas, que levam à aquisição de habilidade ou conjunto de habilidades (SEEFELDT, 1988) e que são sempre da responsabilidade do professor.

\section{Estabilidade e previsibilidade}

A estimação da estabilidade/ "tracking" da coordenação motora grossa por meio do $\gamma$ Foulkes e Davis visa investigar a probabilidade de duas curvas individuais, escolhidas aleatoriamente, serem paralelas 
ao longo do tempo (FoulKes \& Davis, 1981). A sua relevância tem sido apresentada em estudos odontológicos (SChNeiderman \& Kowalski, 1994) e mais recentemente utilizada por Coelho Deus et al. (2008) na pesquisa da estabilidade da coordenação motora grossa em crianças açorianas. Os resultados do $\gamma$ de Foulkes e Davis apresentados na TABELA 2 indicam que em todos os testes não houve qualquer indício de estabilidade em nenhum dos sexos. Os valores mínimos do $\gamma$ para indicar estabilidade relevante iniciam em 0,50 e os encontrados não ultrapassaram 0,20 . O cruzamento das curvas intra-individuais em cada teste pode ser observado graficamente na FIGURA 5. Um padrão semelhante de resultados foi reportado por COELHO Deus et al. (2008), apesar de literalmente mais elevados do que do presente estudo $(\gamma$ máximo de $0,56)$ e o intervalo temporal do estudo ser de quatro anos (seis aos 10 anos). Esses valores reafirmam a ideia de não haver estabilidade na coordenação motora grossa. Por outro lado, WiLLimcZiK (1995) em seu estudo longitudinal com crianças alemãs recorreu a simples técnica de autocorrelação para identificar a estabilidade do coeficiente motor geral, tendo encontrado valores moderados a altos. Essa divergência entre os estudos pode estar associada não só às diferentes técnicas de análise do "tracking", mas também ao intervalo temporal do estudo e ao fato da medida composta de coordenação (coeficiente motor) ser relativamente mais fiável do que os valores individuais dos testes.

A estabilidade entendida como a manutenção da posição relativa do desempenho de cada criança no seio do seu grupo se torna um elemento importante para analisar e discutir a ideia de predição da coordenação ao longo das idades. No entanto, com base nesses resultados fica evidente que as trajetórias individuais apresentam uma forte instabilidade colocando sérios problemas à precisão das previsões. No entanto, ainda assim é possível investigar a estabilidade sob uma ou outra concepção, a do $\kappa$ de Cohen, porque está ancorada na ideia de canais de desempenho.

A estimação da estabilidade/ "tracking" da coordenação motora a partir do $\kappa$ de Cohen visou analisar se o desempenho da coordenação motora grossa dos sete aos 8,5 anos de idade ocorre dentro de canais de desenvolvimento motor específicos. Esse conceito é inicialmente proposto no âmbito da embriologia e da genética por WADDINGTON (1957), e utilizado no campo de investigação do crescimento físico (TANNER, 1990), com fortes evidências empíricas e utilidade no acompanhamento do crescimento de crianças e jovens.
Os resultados do $\kappa$ geral não indicaram qualquer indício de estabilidade da mudança, pois para haver estabilidade moderada era esperado valores acima de 0,40 e os encontrados não ultrapassaram 0,15 tanto para meninas quanto para meninos. Os únicos valores que indicam estabilidade moderada foram os dos testes de saltos monopedais e transposição lateral para as meninas com menor desempenho (ambos com valores de $\kappa=0,48$ no primeiro canal, que diz respeito às meninas com desempenho até o percentil 33). Entende-se que apesar de haver uma estabilidade moderada para as meninas com baixo desempenho nos testes de saltos laterais e transposição lateral, há uma instabilidade na coordenação motora grossa entre os canais ao longo do período de um ano e meio (baixos valores do $\mathrm{K}$ geral).

Com base nesses resultados é possível sugerir que o desenvolvimento coordenativo das melhores crianças aos sete anos podem tanto permanecer como as melhores quanto se enquadrarem como as piores ao longo de um ano e meio em cada um dos testes. Pode-se assim argumentar que a mudança na coordenação motora grossa pode não ocorrer em canais específicos ao longo do tempo, e que o desenvolvimento coordenativo individual se processa de um modo algo errático no intervalo etário estudado.

Essa instabilidade no desenvolvimento da coordenação tem implicações pedagógicas. Por um lado é importante determinar com mais precisão os níveis de prontidão coordenativa iniciais dos alunos e entender o significado da sua variabilidade. Por outro lado é relevante organizar estruturas de aulas com dinamismo suficiente para promover estímulos adequados para o desenvolvimento de todos os alunos para além de monitorar esse processo e analisar os seus resultados. Finalmente, importa salientar a necessidade de mais pesquisa para verificar se esse padrão de instabilidade se verifica ao longo da idade em outras amostras, e buscar esclarecer o porquê de tantas variações interindividuais no desenvolvimento da coordenação motora grossa.

De acordo com os objetivos propostos no presente artigo, pode-se concluir sobre a coordenação motora grossa de crianças de sete aos 8,5 anos de idade que: 1) os quatro testes do KTK apresentaram incremento nos seus valores normativos para ambos os sexos; 2) há uma grande heterogeneidade na mudança ao longo de um ano e meio, não associada ao desempenho coordenativo inicial; 3) a estabilidade interpretada com base nos valores de $\gamma$ Foulkes e Davis foi baixa, indicando uma forte tendência de cruzamentos entre as trajetórias individuais; os valores do $\kappa$ de Cohen também foram baixos, 
sugerindo que o desenvolvimento coordenativo pode não ocorrer em canais específicos ao longo do tempo. Esses resultados reclamam atenção dos professores no sentido de atenderem de modo mais esclarecido às diferenças individuais entre os seus alunos no processo ensino-aprendizagem.

\title{
Nota
}

Projeto financiado em Edital Universal do CNPq $19 \backslash 2004$ - Processo 478202/2004-0 e posteriormente em Edital MCT/ CNPq 15/2007 - Processo 478249/2007-1.

\begin{abstract}
Distinct views on the notion of stability and change in the performance of gross motor coordination

Studies about gross motor coordination in children (GMC) focus on the description of normative values derived from age and sex, and few analyzed the dynamics of change of each child within its group. The goal of the present study is estimate the stability of intra-individual change over inter-individual differences over eighteen months. One hundred and twenty students with seven years old in the city of Muzambinho - MG participated in the study. Data was collected four times, with a six-month interval between each assessment. GMC was evaluated through KTK test battery. Mean values for all tests increased over time, with differences between sex for equilibrium test. The correlation values between initial performance and improvements over eighteen months indicated high heterogeneity. Results of stability estimates were weak for tests. These results were discussed about different trajectories in the development of motor coordination.
\end{abstract}

UnITERMS: Longitudinal study; Inter-individual differences; Intra-individual change; Tracking; Children.

\section{Resumen}

Ángulos diferentes sobre el concepto de estabilidad y cambio en el rendimiento de la coordinación motora gruesa

Las investigaciones sobre la coordinación motora gruesa $(\mathrm{GMC})$ de niños se centran en la descripción de los valores normativos basados en la edad y en el sexo, y pocos estudios analizan la dinámica de cambio en el desempeño de cada niño en relación a su grupo al largo del tiempo. El objetivo de este estudio fue estimar la estabilidad de las diferencias interindividuales en el cambio interindividual de la CMG durante dieciocho meses. Participaron de la investigación 120 estudiantes de siete años de edad de la ciudad de Muzambinho - MG. Cuatro muestras fueron tomadas a intervalos de seis meses. La CMG se evaluó mediante las pruebas del KTK. Los valores medios mostraron aumentos en todas las pruebas, defiriendo entre géneros sólo para el equilibrio a la retaguardia. Los resultados de la correlación entre el rendimiento inicial y el cambio a lo largo del tiempo indicaron una fuerte heterogeneidad. Los resultados de la estabilidad se mostraron débiles. Estos resultados se discuten en términos de diferentes trayectorias de desarrollo de la CMG.

Palabras Clave: Estudio longitudinal; Diferencias interindividuales; Cambio interindividual; Rastreo; Niños. 


\section{Referências}

BOUCHARD, C.; RANKINEN, T. Individual differences in response to regular physical activity. Medicine $\&$ Science in Sports \& Exercise, Madison, v.33, p.446-51, 2001.

CATENASSI, F.Z.; MARQUES, I.; BASTOS, C.B.; BASSO, L.; RONQUE, E.R.V.; GERAGE, A.M. Relação entre índice de massa corporal e habilidade motora grossa em crianças de quatro a seis anos. Revista Brasileira de Medicina do Esporte, São Paulo, v.13, n.4, p.227-30, 2007.

COELHO DEUS, R.K.B.; BUSTAMANTE, A.; LOPES, V.P.; SEABRA, A.F.T.; SILVA, R.M.G.; MAIA, J.A.R. Coordenação motora: estudo de tracking em crianças dos 6 aos 10 anos da Região Autônoma dos Açores, Portugal. Revista Brasileira de Cineantropometria e Desempenho Humano, Florianópolis, v.10, n.3, p.215-22, 2008.

COLLINS, L.M. Analysis of longitudinal data: the integration of theoretical model, temporal design, and statistical model. Annual Review of Psychology, Palo Alto, v.57, p.505-28, 2006.

CONNOLLY, K.J. Skill development: problems and plans: In: CONNOLLY, K. (Ed.). Mechanisms of motor skill development. London: Academic Press, 1970.

D'HONDT, E.; DEFORCHE, B.; GENTIER, I.; DE BOURDEAUDHUIJ, I.; VAEYENS, R.; PHILIPPAERTS, R.; LENOIR, M. A longitudinal analysis of gross motor coordination in overweight and obese children versus normal-weight peers. International Journal of Obesity, London, 2002. doi: 10.1038/ijo.2012.55. [Epub ahead of print].

FOULKES, M.A.; DAVIS, C.E. An index of tracking for longitudinal data. Biometrics, Alexandria, v.37, p.439-46, 1981. GALLAHUE, D.L.; OZMUN, J.C. Compreendendo o desenvolvimento motor: bebês, crianças, adolescentes e adultos. 3. ed. São Paulo: Phorte, 2005.

GRAF, C.; KOCH, B.; FALKOWSKI, G.; JOUCK, S.; CHRIST, H.; STAUENMAIER, K.; BJARNASON-WEHRENS, B.; TOKARSKI, W.; DORDEL, S.; PREDEL, H.G. Effects of a school-based intervention on bmi and motor abilities in childhood. Journal of Sports Science and Medicine, Bursa, v.4, n.3, p.291-9, 2005.

GRAF, C.; KOCH, B.; KRETSCHMANN-KANDEL, E.; FALKOWSKI, G.; CHRIST, H.; COBURGER, S.; LEHMACHER, W.; BJARNASON-WEHRENS, B.; PLATEN, P.; TOKARSKI, W.; PREDEL, H.G.; DORDEL, S. Correlation between BMI, leisure habits and motor abilities in childhood (Chilt-Project). International Journal of Obesity and Related Metabolic Disorders, Hampshire, v.28, n.1, p.22-6, 2004.

HAYWOOD, K.M.; GETCHELL, N. Life span motor development. 4th ed. Champaign: Human Kinetics, 2005.

LANDIS, J.; KOCH, G. The measurement of observer agreement for categorical data. Biometrics, Alexandria, v.33, p.159-74, 1977.

LOPES, V.P.; MAIA, J.A.R. Efeitos do ensino no desenvolvimento da capacidade de coordenação corporal em crianças de 8 anos de idade. Revista Paulista de Educação Física, São Paulo, v.11, n.1, p.40-8, 1997.

LOPES, V.P.; MAIA, J.A.R.; MOTA, J. Aptidóes e habilidades motoras: uma visão desenvolvimentalista. Lisboa: Livros Horizonte, 2000.

LOPES, V.P.; MAIA, J.A.R.; SILVA, R.G.; SEABRA, A.; MORAIS, F.P. Estudo do nível de desenvolvimento da coordenação motora da população escolar (6 a 10 anos de idade) da região autónoma dos Açores. Revista Portuguesa de Ciências do Desporto, Porto, v.3, n.1, p.47-60, 2003.

MAIA, J.A.R.; BEUNEN, G.; LEFEVRE, J.; CLAESSENS. Testes empíricos a formulações desenvolvimentistas: um estudo centrado na modelação da estrutura de covariância. Revista Paulista de Educação Física, São Paulo, v.12, n.2, p.160-80, 1998.

MAIA, J.A.R.; GARGANTA, R.M.; SEABRA, A.; LOPES, V.P.; SILVA, S.; MEIRA JUNIOR, C.M. Explorando a noção e significado de tracking: um percurso didáctico para investigadores. Psicologia.pt: o Portal dos Psicólogos, Lisboa, p.1-23, 2007. Disponível em: <http://ww.psicologia.pt/artigos/textos/A0348.pdf>.

MALINA, R.M.; BOUCHARD, C.; BAR-OR, O. Growth, maturation, and physical activity. Champaign: Human Kinetics, 2004.

MORTIMER, J.T.; FINCH, M.D.; KUMKA, D. Persistence and change in development: the multidimensional selfconcept. In: LIFE-SPAN development and behavior. New York: Academic Press, 1982. v.4, p.263-313.

NESSELROADE, J.R. Interindividual differences in intraindividual change. In: COLLINS, L.M.; HORN, J.L. (Eds.). Best methods for the analysis of change: recent advances, unanswered questions, future directions. Washington: American Psychological Association, 1991. p.92-105.

PANAGOPOULOU, V.; NAKOU, I.; GIANNAKOULIA, V.; SERBEZIS, V. Evaluation of motor coordination in early school aged children. European Psychomotricity Journal, Thrace, v.1, n.1, p.36-9, 2008. 
ROBERTON, M.A.; HALVERSON, L.E. Developing children - their changing movement: a guide for teachers. Philadelphia: Lea \& Febiger, 1984.

SCHILLING, F.; KIPHARD, E.J. Körperkoordinationstest für kinder, KTK. Weinheim: Beltz Test Gmbh, 1974.

SCHILLING, V. F.; KIPHARD, E.J. The body coordination test (BCT). Journal of Physical Education and Recreation, Reston, v.47, n.4, p.37, 1976.

SCHNEIDERMAN, E.; KOWALSKI, C.J. Analysis of longitudinal data in craniofacial research: Some strategies. Critical Reviews in Oral Biology and Medicine, Boca Raton, v.5, p.187-202, 1994.

SEFELDT, V. The concept of readiness applied to motor skill acquisition. In: SMOLL, F.; MAGILL, R.; ASH, M. (Eds.). Children in sport. Champaign: Human Kinetics, 1988.

TANI, G. Educação física na pré-escola e nas quatro primeiras séries do ensino de primeiro grau: uma abordagem de desenvolvimento. Kinesis, Santa Maria, v.3, n.1, p.19-41, 1987.

TANI, G.; GAYA, A.; MARQUES, A.T. Desporto para crianças e jovens: razões e finalidades. Porto Alegre: Universidade Federal do Rio Grande do Sul, 2004.

TANI, G.; MANOEL, E.J.; KOKUBUN, E.; PROENÇA, J.E. Educação física escolar: fundamentos de uma abordagem desenvolvimentista. São Paulo: Editora da Universidade de São Paulo, 1988.

TANNER, J.M. Foetus into man: physical growth from conception to maturity. Cambridge: Harvard University Press, 1990.

VALDIVIA, A.B.; CARTAGENA, L.C.; SARRIA, N.E.; TÁVARA, I.S.; TEIXEIRA, A.F.; DA SILVA, S.R.M.G.; MAIA, J.A.R. Coordinación motora: influencia de la edad, sexo, estatus socio-económico y niveles de adiposidad en niños peruanos. Revista Brasileira de Cineantropometria e Desempenho Humano, Florianópolis, v.10, n.1, p.25-34, 2008. WADDINGTON, C.H. The strategy of the genes. London: Allen \& Unwin, 1957.

WILLIMCZIK, K. Development of motor control capability (body coordenation) of 6-to-10-years-old children: results of a longitudinal study. In: OSTYN, M.; BEUNEN, G.; SIMONS, J. (Eds.). Kinanthropometry II. Baltimore: University Park Press, 1980.

\begin{tabular}{r|l} 
ENDEREÇO & \\
Go Tani & \\
Escola de Educação Física e Esporte- USP & Recebido para publicação: 13/03/2009 \\
Av. Prof. Mello Moraes, 65 & Revisado: 09/05/2012 \\
05508-030 - São Paulo - SP - BRASIL & Aceito: 25/05/2012 \\
e-mail: gotani@usp.br & \\
&
\end{tabular}

